\title{
Evaluation of an evidence based quality improvement innovation for patients with musculoskeletal low back pain in an accident and emergency setting
}

\author{
Tara Potier, Emily Tims, Cherry Kilbride, Khadija Rantell \\ Physiotherapy Division, Department of Clinical Sciences, College of Health and Life Sciences, Brunel University London, UK and UCL Institute \\ of Neurology, Education Unit, Queen Square, London
}

\begin{abstract}
We conducted a five stage pilot study which initially consisted of a review of 75 case notes of people attending an emergency department (ED) in an inner London Teaching Hospital with musculoskeletal (MSK) low back pain (LBP). This review highlighted inconsistencies in how they were assessed and managed across and within different staff groups. We found patient documentation was often incomplete and that a biomedical model approach to the management of these patients was common.
\end{abstract}

As a result, four further stages in the project were conducted. Our primary aim was to evaluate the impact of implementing a locally developed quality improvement intervention for the assessment and treatment of MSK LBP in this ED. Secondary aims were to explore the user experience of the new pathway, measured by the patient experience questionnaire (PEQ), and any associated health economic costs of changes in practice. The quality improvement intervention consisted of an evidence based low back pain pathway (EBLBPP), a staff educational program, and a patient education booklet.

We undertook a retrospective baseline audit of 100 clinical records of patients was undertaken prior to the instigation of the quality improvement intervention, and four months post implementation. The pre-defined variables of interest were: documentation of the case history, examination, classification of back pain (and if correct), prescribed management and if the documentation was compliant with medico-legal standards. All patients in the study were sent a PEQ to complete and return in a self-addressed envelope. Estimated health costs associated with each patient episode of care were calculated including re-attendance episodes for any people presenting with MSK LBP within a four week period.

\begin{abstract}
There was a significant improvement in all areas evaluated post implementation in all groups (simple, referred and simple, referred and serious spinal pathology combined). In particular; screening for red flags (22\%) and biopsychosocial factors (29\%), as well as noting the prevalence of previous symptoms (44\%), observation of the painful area (57\%), and analysis classification (59\%) at a at $95 \%$ confidence interval (Cl). In terms of management, an increase in adherence to the analgesic ladder, patients receiving reassurance and appropriate referral back to their GP's increased $45 \%, 23 \%$ and $44 \%$ at $95 \% \mathrm{Cl}$ respectively. Unfortunately, there was insufficient data to draw any meaningful conclusions from the patient experience data due to a low response rate.
\end{abstract}

In conclusion, the introduction of the EBLBPP, patient education leaflet and teaching training for staff involved in the treatment of MSK LBP patients has improved the quality and consistency of the documented assessment and subsequent management of MSK LBP patients.

\section{Problem}

MSK conditions are increasing and accounting for approximately $£ 10$ billion of the National Health Service's (NHS) £110 billion budget making it the third most expensive specialty after mental health and cardiac. MSK LBP is the most prevalent MSK disorder affecting nearly everyone at some point in time, about 4-33\% at any given time [1]. ED's are often the first point of contact for these patients. Within the ED in this study there were anecdotal inconsistencies reported in the management of MSK LBP. This led to an informal review of patients' notes who attended the ED with MSK LBP which confirmed these suspicions. As a result of these findings, this five phase study was designed and implemented.

\section{Background}

MSK LBP affects approximately one-third of the UK adult population each year, accounting for about $30 \%$ of GP consultations,[2] consuming over $20 \%$ of the UK's total health expenditure.[3] Back pain is a challenging clinical area for clinicians; often lacking clarity of diagnosis and treatment. One year after a first episode of back pain, $62 \%$ of people report that they still have pain and $16 \%$ of those initially unable to work are still not back in employment.[4] As such, chronic MSK LBP can result in persistent disability, lower quality of life and reduced capacity to work.[5]

Early intervention for back pain is key to decreasing chronicity and achieving a speedy and sustained recovery.[6] Advice, pain relief, 
and rapid rehabilitation through self help, staying active, and supportive physical therapies is also valuable.[6] One of the biggest indicators for an individual developing chronicity are biopsychosocial factors, referred to as "yellow flags" which affect a patients' emotional and behavioural response to pain [7].

Yellow flags include: emotional responses, pain behaviours, health beliefs, appraisals and judgements; they are especially influential in the transition between acute and chronic problems.[8] Evidence suggests that identification of yellow flags in patients' results in better outcomes when compared to patients who had these factors ignored.[9] Assessment of yellow flags at the patient's initial presentation is therefore highly recommended. [5,10-14]

Reassurance and education for patients presenting with MSK LBP in primary care has also been shown to promote the recovery process.[15]

In addition, presenting LBP should be categorised into simple MSK LBP, referred MSK LBP and serious spinal pathology as each category requires a specific baseline of clinical assessment and management.[5,10-14]

Attendance at EDs has increased by over $50 \%$ in the last decade.[16] There are no studies to the author's knowledge looking at treatment of MSK LBP within the ED environment. The ED, in this quality improvement project, sees approximately 100 patients a month with MSK LBP. Most commonly these patients are treated within a biomedical model, where the focus of attention is on the pathology. This approach has been linked with an increased risk of chronicity[17] and importantly can affect how the patient responds to any subsequent treatment.[18] To improve outcomes for this patient group the need for a change in practice was apparent.

\section{Baseline measurement}

The first data collection point (pilot study) consisted of a review of 75 case notes of patients presenting to the ED of a larger inner city teaching hospital with MSK LBP showed variation in the process of assessment and management within and across different clinical staff; namely the ED doctors, general practitioners (GPs) and an extended scope physiotherapist (ESP). Differences seen included:

- Variation in the content of assessment and management

- Patients investigated despite there being no identified clinical need, eg lumbar spine $x$ rays $(n=16,21 \%)$

- Unnecessary referrals to secondary care services, eg neurosurgery despite no indications for surgery $(n=2,3 \%)$

- Re-attendance to the ED with MSK LBP within a four week period $(n=4 ; 5 \%)$

- Non-documentation of red flags which indicate serious pathology such as a possible tumour $(12 \% ; n=9)$.

All case notes showed a biomedical model approach to patient care; this is where the primary focus is on the presenting pathology.
There was no indication that any psychosocial factors had been considered, such as the patients' attitude to their pain or support mechanisms at home. Few case notes documented if any advice and or reassurance was given to the patients as part of their care. Factors such as these are known to be important, as the biomedical model approach to low back pain of MSK origin has been linked to an increased risk of the patient developing chronic pain. [6,8]

In addition, this note review raised an important concern regarding the standard of assessment and documentation. Notes were not always compliant with the international guidelines for the management of MSK LBP [5,10-14] and thereby suggestive that the management of these patients may have been incomplete, and not in line with best practice.

See supplementary file: ds5758.doc - "Appendix 1: EBLBPP"

\section{Design}

This study consisted of five phases: first data collection point (notes review), developmental, second data collection point (pre implementation), implementation, and third data collection point (post implementation). The primary aim of this pilot study was to evaluate the impact of implementing a locally developed quality improvement intervention for the assessment and treatment of MSK LBP in this ED. This quality intervention comprised of an EBLBPP, a staff education program and education booklet for patients. Secondary aims were to explore the user experience of the new pathway, measured by the PEQ, and any associated health economic costs of changes in practice.

\section{Strategy}

Developmental phase:

A critical review of the current literature and guidelines was undertaken to inform the development of:

- Three audit tools for assessing the clinical documentation for the respective clinical classifications of MSK LBP: simple; referred and serious spinal pathology

- The development of an EBLBPP (appendix 1)

- A patient education booklet

- Staff educational program.

The quality improvement intervention consisted of:

- The introduction of the EBLBPP

- A staff educational program, and

- Patient education booklet.

The audit tools, EBLBPP, and patient education booklet were based 


\section{BMJ Quality Improvement Reports}

on international guidelines [5,10-14] and agreed by an Urgent Care Centre (UCC) General Practitioner (GP), ED Consultant and an ESP specialising in acute MSK medicine. The audit tools and EBLBPP was piloted on the 75 sets of notes used for the informal review. The inter-rater and intra-rater reliability of the audit tool was 0.85 or higher and 0.90 or higher respectively between the ESP and senior independent physiotherapy researcher.

The three audit tools collected the following information:

- Subjective history, eg where the pain is, how long they have had their pain

- Objective assessment findings e.g. range of motion of the spine, neurological examination

- Investigations ordered, eg $x$ rays or scans

- Treatments delivered, eg back care advice, medication

Audit data was bench marked against internationally accepted guidelines for MSK LBP to identify areas of improvement and potential cost savings, including the use of the World Health Organisation analgesic ladder.[5,10-14,19]

Evaluation of patient experience:

The PEQ is an 18 item self-reported reliable and validated tool designed to measure the patient experience of treatment received.[20] No personal identifying data is collected.

Evaluation of cost- health economic data:

The cost of interventions, diagnostic tests and onward referrals associated with the treatment of MSK LBP were calculated by the Trust's finance department.

Second data collection point (pre-implementation phase):

Recruitment: A daily report was generated from the ED computerised record system to identify patients who had been seen that day with symptoms of MSK LBP. A senior independent physiotherapist researcher then screened the notes to ensure the patient selected fulfilled the inclusion criteria (see section below). All patient information was anonymised in order to comply with the Data Protection Act 1998. Once 100 consecutive ED patient attendances for MSK LBP, who fulfilled the inclusion criteria, were reached the daily computer reports were stopped. Ninety-two patients did not meet the inclusion criteria, which were:

- 18 years of age and over

- Presented with any of the following symptoms: low back pain, back pain, acute back pain, red flags, spinal fracture, cauda equine syndrome, spinal infection.

All scanned documentation associated with each episode of care was analysed including: discharge letters, investigations and onward referral letters using the audit tool for the patient's classification of MSK LBP. The cost of the episode of care was calculated using this information which informed the health economic analysis for this phase.

The 100 patients meeting the inclusion criteria for the study were posted a participant information sheet and PEQ within three days of attending the ED. A pre-paid self-addressed envelope was provided.

Health professionals involved in the study were all staff responsible for treating MSK LBP patients in the ED which consisted of UCC GPs, ED consultants, ED registrars, foundation year 2's (FY2), specialist trainee 1's (ST1) and 2's (ST2), and an ESP.

Data analysis: Data from the simple, referred and serious spinal pathology audit tools were analysed to compare pre and post implementation results. The simple and referred patient subgroups were initially analysed separately; the numbers of people presenting within the serious category were too small to undertake a separate analysis. A final analysis was performed combining the simple, referred and serious patients. Numerical data were summarised using mean and standard deviation, or median and interquartile range. Categorical data were summarised using count and percentages. We used the normal approximation to the binomial distribution to compare the proportion of success in areas evaluated before and after implementation of quality improvement program.

Intervention phase: During this phase all staff involved in the treatment of patients with MSK LBP in the ED were targeted for training. This took the form of a one hour mandatory session in best practice treatment for patients with MSK LBP. Content included how to use the new EBLBPP for patients with MSK LBP and the importance of providing the new patient leaflet. This leaflet incorporated advice and education on the patients' current episode of MSK LBP as well as what action to take should the pain re-occur

Third data collection point (post implementation phase): The same evaluation measures were used as during the second data collection point (notes analysis, patient's satisfaction survey and an economic health analysis) on 100 MSK LBP ED consecutive attendances. Ninety-five sets of patient notes did not meet the inclusion criteria and were excluded.

\section{Results}

Case notes were analysed at two time points: second data collection point (pre implementation) and four months post implementation (third data collection point). 100 sets of notes were analysed at both stages and categorised into simple MSK LBP, referred MSK LBP and serious spinal pathology. The distribution is shown in figure 1 and the patient demographics in figure 2 .

Figure 3 shows the percentage of success area evaluated before and after implementation of quality improvement intervention. Estimates of the comparisons of the proportion of success in area evaluated before and after implementation of the quality improvement intervention are provided in table 1. Summary of key 


\section{BMJ Quality Improvement Reports}

features by type of MSK LBP are described below.

Simple MSK LBP:

Subjective History: The documentation of all case history variables for patients presenting with simple MSK LBP (ie red flags, location of pain, frequency of symptoms, duration of symptoms, previous symptoms, yellow flags assessed, and response to previous treatment) improved significantly between pre and post implementation. Key areas of assessment, such as red flags and biopsychosocial factors demonstrated a significant improvement of $27 \%(11 \%, 42 \%)$ and $34 \%(21 \%, 47 \%)$ at $95 \%$ confidence interval (Cl) respectively. The largest percentage changes at $95 \% \mathrm{Cl}$ were seen in notation of previous symptoms $(45 \%(30 \%, 60 \%))$ and frequency of symptoms $(41 \%(26 \%, 56 \%))$.

Examination: The largest impact of the intervention program was seen in in the recorded observation of the painful area $157 \%(43 \%$, $70 \%$ ) at $95 \% \mathrm{Cl})$, range of motion $(29 \%(12 \%, 38 \%)$ at $95 \% \mathrm{Cl})$, and palpation of the painful area $(25 \%(12 \%, 38 \%)$ at $95 \% \mathrm{Cl})$. Changes were also seen to a lesser extent in contralateral straight leg raise $(\mathrm{SLR})(24 \%(9 \%, 39 \%)$ at $95 \% \mathrm{Cl})$ and Babinski $(19 \%$ $(8 \%, 29 \%)$ at $95 \% \mathrm{Cl})$.

Classification: Pre-implementation, a diagnosis was given for $39 \%$ $(n=23 / 59)$ of patients, of which one-quarter $(n=5 / 23)$ were correct. Post-implementation a diagnosis was given to $99 \%(n=73 / 74)$ of patients, of which three-quarters $(n=56 / 73$ ) were correct.

Investigations: There was a decrease in all investigation except $\mathrm{X}$ ray done which increased by $3 \%$ post implementation.

Management: Significant improvements between the second and third data collection points were seen in the use of the analgesic ladder $(49 \%(36 \%, 62 \%)$ at $95 \% \mathrm{Cl})$ and for patients being educated and reassured $(18 \%(4 \%, 31 \%)$ at $95 \% \mathrm{Cl})$. A significant increase was noticed for patients being referred back to their GP post implementation of $49 \%$ (34\%, 64\%), including a rise of $50 \%$ $(35 \%, 65 \%)$ at $95 \% \mathrm{Cl}$ in when this was the appropriate.

\section{Referred MSK LBP:}

Subjective history: All documentation of required case history variables showed a percentage improvement between the phases. The largest impact was seen in assessment of red flags $(24 \%$ $(10 \%, 37 \%)$ at $95 \% \mathrm{Cl})$, previous symptoms $(51 \%(30 \%, 71 \%)$ at $95 \% \mathrm{Cl})$ and response to treatment $(42 \%(19 \%, 65 \%)$ at $95 \% \mathrm{Cl})$.

Examination: Assessment of all variables post implementation showed a substantial percentage increase: observation of the painful area $(57 \%(39 \%, 74 \%)$ at $95 \% \mathrm{Cl})$, ROM $(56 \%(37 \%, 76 \%)$ at $95 \% \mathrm{Cl})$, palpation of the painful area $(32 \%(17 \%, 46 \%)$ at $95 \%$ Cl), ipsilateral SLR $(35 \%(13 \%, 58 \%)$ at $95 \% \mathrm{Cl})$, contralateral SLR $(36 \%(13 \%, 60 \%)$ at $95 \% \mathrm{Cl})$, dermatomes $(42 \% 23 \%, 61 \%)$ at $95 \% \mathrm{Cl})$, myotomes $(25 \%(2 \%, 47 \%$ at $95 \% \mathrm{Cl}))$, reflexes $(29 \% 7 \%$, $50 \%)$ at $95 \% \mathrm{Cl})$ and Babinski $(27 \% 5 \%, 49 \%)$ at $95 \% \mathrm{Cl})$.

Classification: A diagnosis was given in 54\% (36\%, 71\%) more cases post implementation, however, this diagnosis was only deemed accurate in $6 \%(-7 \%, 30 \%)$ of cases at $95 \% \mathrm{Cl}$.

Investigations: There was an increase in all investigations performed but all of these were deemed to be indicated.

Management: Use of the analgesic ladder, $57 \%(n=20 / 35)$, and provision of education and reassurance $14 \%(n=3 / 38)$ significantly improved to $100 \%(n=25 / 25)$ and $44 \%(n=11 / 25)$ of cases respectively. Analysis of data on the management of these patients suggested only referrals back to the GP and whether these referrals were indicated varied between the two time periods showing a percentage improvement of $29 \%(5 \%, 53 \%)$ and $33 \%(9 \%, 56 \%)$ at $95 \% \mathrm{Cl}$ respectively.

Serious spinal pathology: Due to the small number of patients $(n=3$ pre implementation and $n=2$ post implementation), no subgroup analysis was possible of this cohort. Observational analysis of the data sets indicated the correct pathway had been followed in all cases.

Combined analyses for simple MSL LBP, referred MSK LBP and serious spinal pathology back pain: The combined results for simple, referred and serious MSK LBP suggest all case history variables, examination and the 3 classifications variables were significantly more frequently documented post implementation than pre implementation. Of note, red flags and biopsychosocial screening increased by $22 \%(10 \%, 34 \%)$ and $29 \%(18 \%, 40 \%)$ at $95 \% \mathrm{Cl}$ respectively. The largest impact was seen notation of previous symptoms $(44 \%(31 \%, 56 \%)$ at $95 \% \mathrm{Cl})$ and response to previous symptoms $(38 \%(25 \%, 51 \%)$ at $95 \% \mathrm{Cl})$.

During examination the largest impact was seen observation of the painful area at $57 \%(46 \%, 68 \%)$ improvement at $95 \% \mathrm{Cl}$. A $59 \%$ $(49 \%, 69 \%)$ was seen in classification of the patients with $33 \%$ $(16 \%, 51 \%)$ improvement at $95 \% \mathrm{Cl}$ in this classification being correct. Four $\mathrm{X}$ rays were ordered which were not indicated post implementation compared to one pre implementation.

Results in the management category showed improvements in the analgesic ladder being followed improved by $45 \%$ (35\%, 55\%) and the provision of education and reassurance rose from $11 \%$ $(n=11 / 97)$ pre implementation to $34 \%(n=34 / 99)$ post implementation at $95 \% \mathrm{Cl}$.

Referrals back to the GP and that referral being clinically indicated significantly improved by $42 \%(29 \%, 54 \%)$ and $44 \%(31 \%, 56 \%)$ at $95 \% \mathrm{Cl}$ respectively.

Economic health costs: No significant differences between the second and third data collection points were found between the number of investigations ordered ( $x$ ray, urine analysis, and basic blood screen), the rates of referrals to secondary care or physiotherapy or the number of people admitted to the overnight ED ward. Results did highlight a number of other unnecessary investigations were still being requested. This was most prominent in the simple MSK LBP group where pre-implementation there was $£ 50.15$ of unnecessary expense, whereas post implementation this 
increased to £202.29. This unexpected finding from the implementation of the new pathway appeared to be largely due to $x$ rays being ordered that from the documented case history were not clinically indicated as necessary. However, a fall from in the number of discharge letters to GP's ( $n=5$ to $n=1$ ) that requested further investigations that were not indicated was noted.

Re-attendance rates: There was only one patient who re-attended within one month pre-implementation, compared to eight returners during the post phase.

PEQ data: The respondent return rate of the PEQ was very low, $n=6 / 100$ pre implementation and $n=4 / 100$ post implementation, thus precluding statistical analysis. Visual analysis of the PEQ data returned showed an improvement in all subsections of patient satisfaction except "communication barriers", where patients reported still not feeling involved in decision making about their care.

See supplementary file: ds5844.docx - "Figures 1, 2, 3 and Table 1"

\section{Lessons and limitations}

As a consequence of the first data collection that was performed this study was developed. Between the first and second data collection points the study evolved considerably and therefore making valid comparisons between the first, second and third data collection points was not possible, limiting the data analysis to between second and third data collection points only.

Ideally consistent ED staff members would have been maintained throughout the life of the project. Due to the very nature of an ED this was not possible because of the transient and rotational nature of the staff members that is usual in such departments. However, when new staff were inducted into the department the use of the EBLBPP and patient education sheets were highlighted, therefore minimising the disruption. If possible they were also asked to attend the staff education session. In addition, the ESP role was discontinued during the project which resulted in responsibility for compliance with the project being transferred to two ED FY2's and the education program was delivered by an ED registrar. The responsibility for enhancing the progress made with this study will be transferred annually to two allocated ED FY2's who will repeat the project at as part of their responsibilities during their time within the ED.

PEQ respondent numbers were lower than expected resulting in making meaningful conclusions from the data impossible. Were the study to be repeated immediate feedback post consultation via portal stands, hand held devices or face to face interviews would be proposed.

This study was undertaken with the long term aim to extend the quality improvement innovations in this project to include patients presenting with MSK LBP in primary care as well as those in secondary care. This would enable patients to be assessed and tracked across both environments. Improvements to data analysis would be made to include a cost analysis of medication and explore ways to better engage patients in providing feedback on their experiences.

\section{Conclusion}

All cohorts of MSK LBP showed an improvement as a result of implementation of an EBLBPP, patient education leaflet and educational program in the documented history, assessment and management variables of patients who attended the ED with MSK LBP. These changes in practice are suggestive that the care provided aligns more with best practice and is less dependent on which clinician assesses the patient. Of note, red flags and biopsychosocial screening, as well as, patient education and reassurance are key considerations for accurate long term management of MSK LBP patients.

Although not statically significant, the health economic costs of implementing the quality improvement interventions (EBLBPP, patient education leaflet, and staff education program) show that there is still a learning need for the department with regards to ordering unnecessary investigations, especially in patients with simple MSK LBP. These learning needs for the ED will be addressed through further staff education.

The number of re-attendances increased from one to eight after the implementation stage. Further review of these notes revealed valid reasons for these patients seeking further medical attention, however not in the ED. For example, two of the patients had a significant increase in their pain. It can by hypothesised the rise in re-attendances post implementation is reflective of patients receiving a higher quality of service and satisfaction in the service than they previously did. Before this project, patients may have sought medical attention from elsewhere after attending this ED but now they may be returning to where they feel they have received the best treatment. Unfortunately, the numbers of respondents to the PEQ was too small to provide any further insights as to why this maybe the case. Nonetheless, the number of re-attendances post implementation has highlighted a need for further development of the educational resources and patient information leaflet to help signpost patients to seek help first in primary care.

Informal feedback from ED staff suggests the EBLBPP and patient education leaflet is quick and easy to use, thus indicating they are time efficient to use. In addition, the increased number of patients being appropriately recommended to see their GP points to an increase in holistic management by crossing into primary care. It is not possible to ascertain whether such changes as these are as a result of an improved awareness of the potential implications of biopsychosocial factors, (as highlighted with the increase in yellow flags being assessed), this requires a more in-depth examination of practice and follow up of patients across the pathway.

In conclusion, the introduction of this quality improvement intervention (EBLBPP, patient education leaflet, and teaching sessions) has significantly improved the quality and consistency of the documented assessment and management of MSK LBP patients. Further work is required to track patients through primary and other secondary care providers to gauge the full impact of this 


\section{BMJ Quality Improvement Reports}

quality innovation.

\section{References}

1. Woolf A, Pfleger B. Burden of Major Musculoskeletal conditions. Bulletin of the World Health Organisation. 2013;81:646-56.

2. Macfarlane GJ, Jones GT, Hannaford PC. Managing low back pain presenting to primary care: where do we go from here? Pain 2006 June;122(3):219-22.

3. Maniadakis N, Gray A. The Economic Burden of Back Pain in the UK. Pain 2000 Jan;84(1)95-103.

4. Hestbaek L, Leboeuf-Yde C, Manniche C. Low back pain: what is the long-term course? A review of studies of general patient populations. Eur Spine J 2003 Apr;12(12):149-65.

5. McGrail M, Lohman W, Gorman R. Disability prevention principles in the primary care office. Am Fam Physician 2001 Feb 15;63(4):679-84.

6. Black C. Working for a healthier tomorrow. London: The Stationery Office. 2008.

7. Engel GL. The need for a new medical model: a challenge for biomedicine. Science 1997 Apr 8;196(4286):129-36.

8. Linton SJ. A review of psychological risk factors affecting back and neck pain. Spine 2000 May 1;25(9):1148-56.

9. Nicholas MK, Linton SJ, Watson PJ, Main CJ, the "Decade of the Flags" Working Group. Early Identification and Management of Psychological Risk Factors ("Yellow Flags") in Patients with Low Back Pain: a reappraisal. Phys Ther 2011 May;91(5);737-53.

10. Royal College of General Practitioners. Clinical Guidelines for the Management of Low Back Pain. London: Royal College of General Practitioners. 1994.

11. NHS Centre for Reviews and Dissemination. Acute and chronic low back pain. Effective Health Bulletin. 2000; 6(5).

12. Accident Compensation Corporation. New Zealand Acute Low back pain guide. Wellington, New Zealand: Accident Compensation Corporation. 2004.

13. Chou R, Qaseem A, Snow V, Casey D, Cross T, Shekelle $P$, et al. Diagnosis and Treatment of Low Back Pain: A Clinical Practice Guideline from the American College of Physicians and the American Pain Society. Ann Intern Med 2007 Oct 2;147(7):478-91.

14. Tulder M, Becker A, Bekkering T, Breen A, Carter A, Gil del Real M. et al European Guidelines for the management of acute nonspecific low back pain in primary care. Eur Spine $J$ 2006:15 Suppl 2:S169-91.

15. Pincus $T$, Holt N, Vogel S, Underwood M, Savage R, Walsh $D A$, et al Cognitive and affective reassurance and patient outcomes in primary care: a systematic review. Pain 2013 Nov;154(11):2407-16.

16. Health and Social Care Information Centre. Hospital Episode Statistics: Accident and Emergency Hospital Attendances 2012-2013. Leeds: Health and Social Care Information Centre. 2014.

17. Linton SJ, Andresson T. A randomized controlled design superimposed on treatment as usual was used to compare the effects of a cognitive-behaviour intervention aimed at preventing chronicity with two different forms of information.
Spine 2000 Nov 1;25(21):2825-31.

18. Skelton AM, Murphy EA, Murphy RJ, O'Dowd TC. Patients' views of low back pain and its management in general practice. Br J Gen Pract 1996 Mar;46(404):153-6.

19. World Health Organisation. Cancer Pain Relief: a guide to opoid availability. Geneva: World Health Organisation. 1997.

20. Steine, S, Finset $A$ and Laerum $E$ A new, brief questionnaire $(\mathrm{PEQ})$ developed in primary care for measuring patients' experience of health interaction, emotion and consultation outcome. Fam Pract 2001 Aug;18(4):410-7.

\section{Declaration of interests}

A research grant was awarded to the project from the NHS Trust's Research and Development Department for £11, 252.95.

\section{Acknowledgements}

Dr Jonathan Costello, Dr Farzad Entikabi, and Paul Bassett.

\section{Ethical approval}

This project was approved by a University Ethics Committee and had site-specific approval from the research and development department. 